Supporting Information

\title{
Growing Pt Nanowires as a Densely Packed Array on Metal Gauze
}

\author{
Eric P. Lee ${ }^{\dagger}$, Zhenmeng Peng ${ }^{\ddagger}$, David M. Cate, ${ }^{\dagger}$ Hong Yang, ${ }^{\ddagger}$ Charles T. Campbell, ${ }^{\dagger}$ and
} Younan $\mathrm{Xia}^{\dagger, *}$

${ }^{\dagger}$ Department of Chemistry

University of Washington, Seattle, Washington 98195-1700

${ }^{\ddagger}$ Department of Chemical Engineering

University of Rochester, Rochester, New York 14627-0166

*Corresponding author.E-mail: xia@chem.washington.edu 


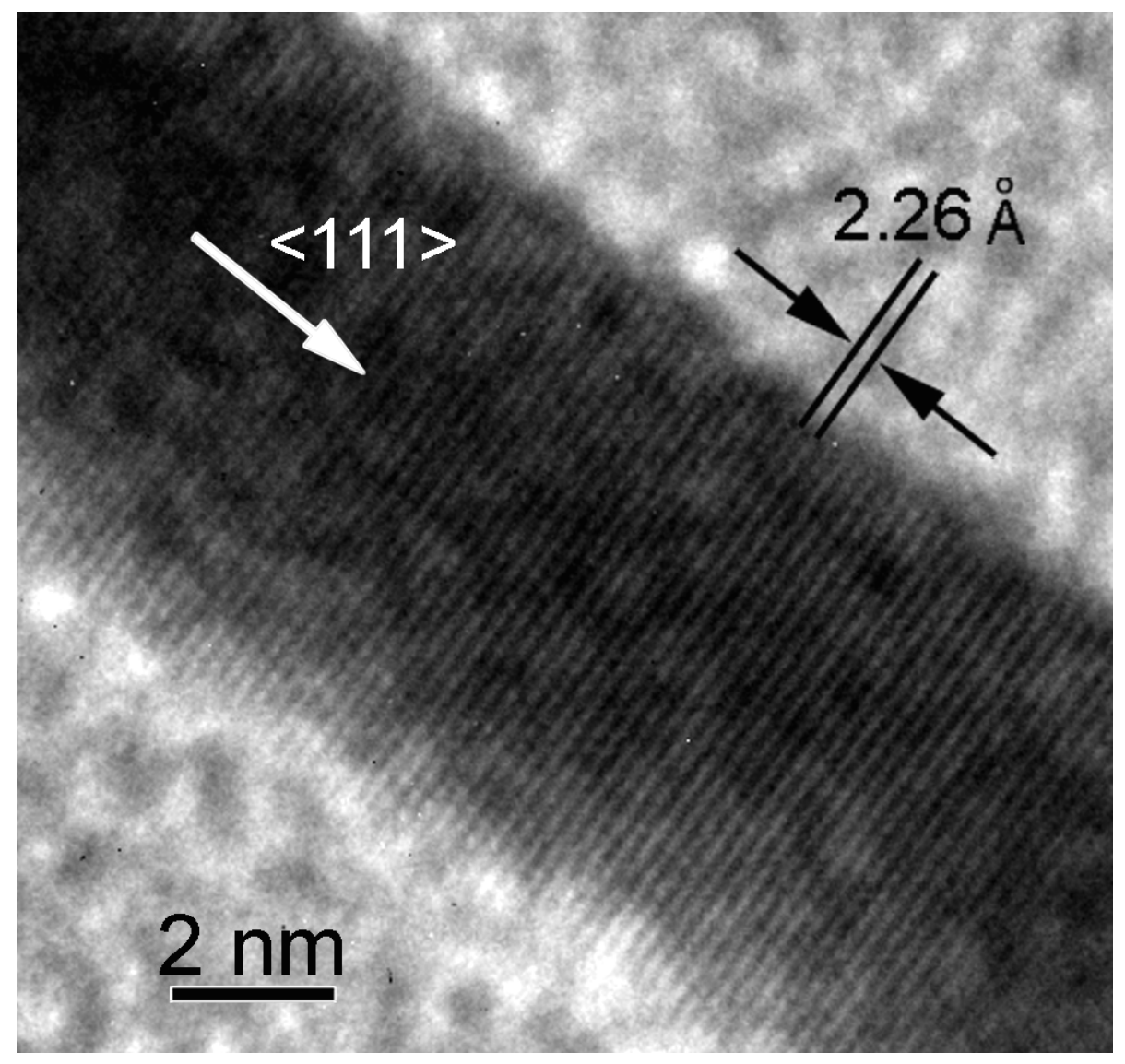

Figure S1. HRTEM image of an individual Pt nanowire. The image shows that the nanowire is single crystalline. The fringe spacing of $2.26 \AA$ corresponds to the interplanar separation between the $\{111\}$ planes, implying that the growth direction of the nanowire is along the $<111>$ axis. The $2.26 \AA$ lattice spacing between the $\{111\}$ planes also indicates that the nanowire was crystallized in a face-centered cubic $(f c c)$ structure. 

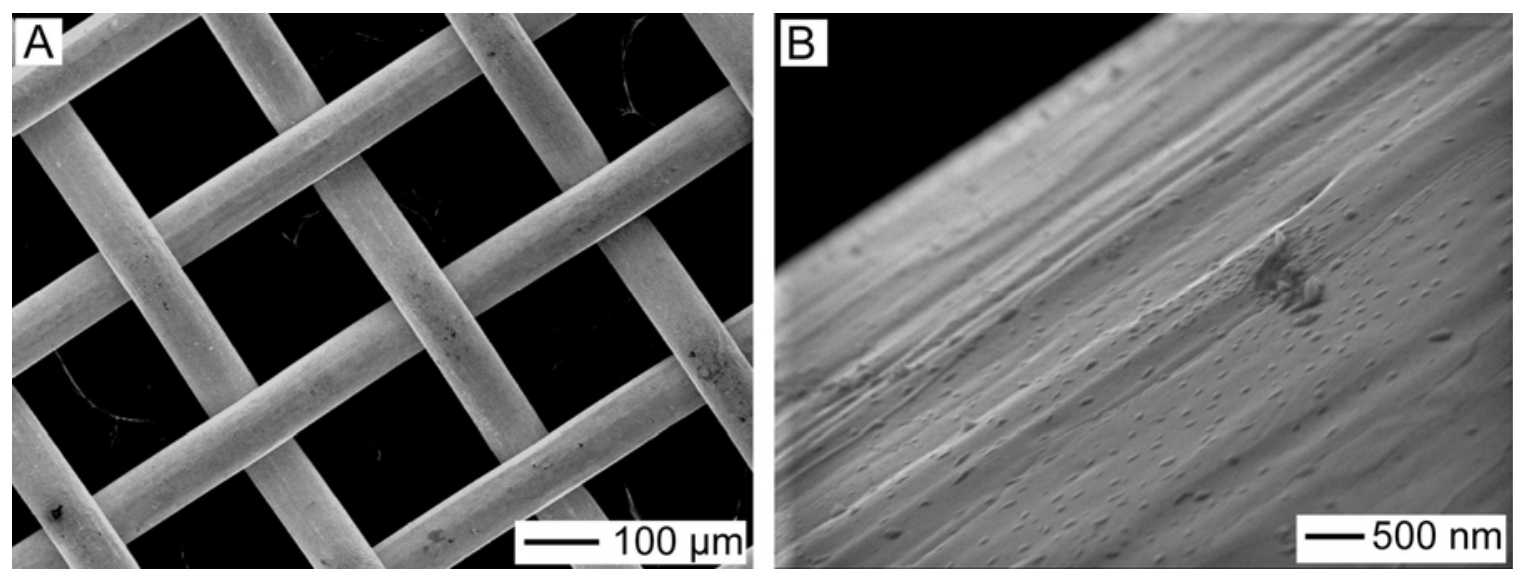

Figure S2. (A) SEM image of pristine Pt gauze. (B) Enlarged SEM image of the same Pt gauze, showing a highly rough surface that facilitates the nucleation and growth of Pt nanowires. 

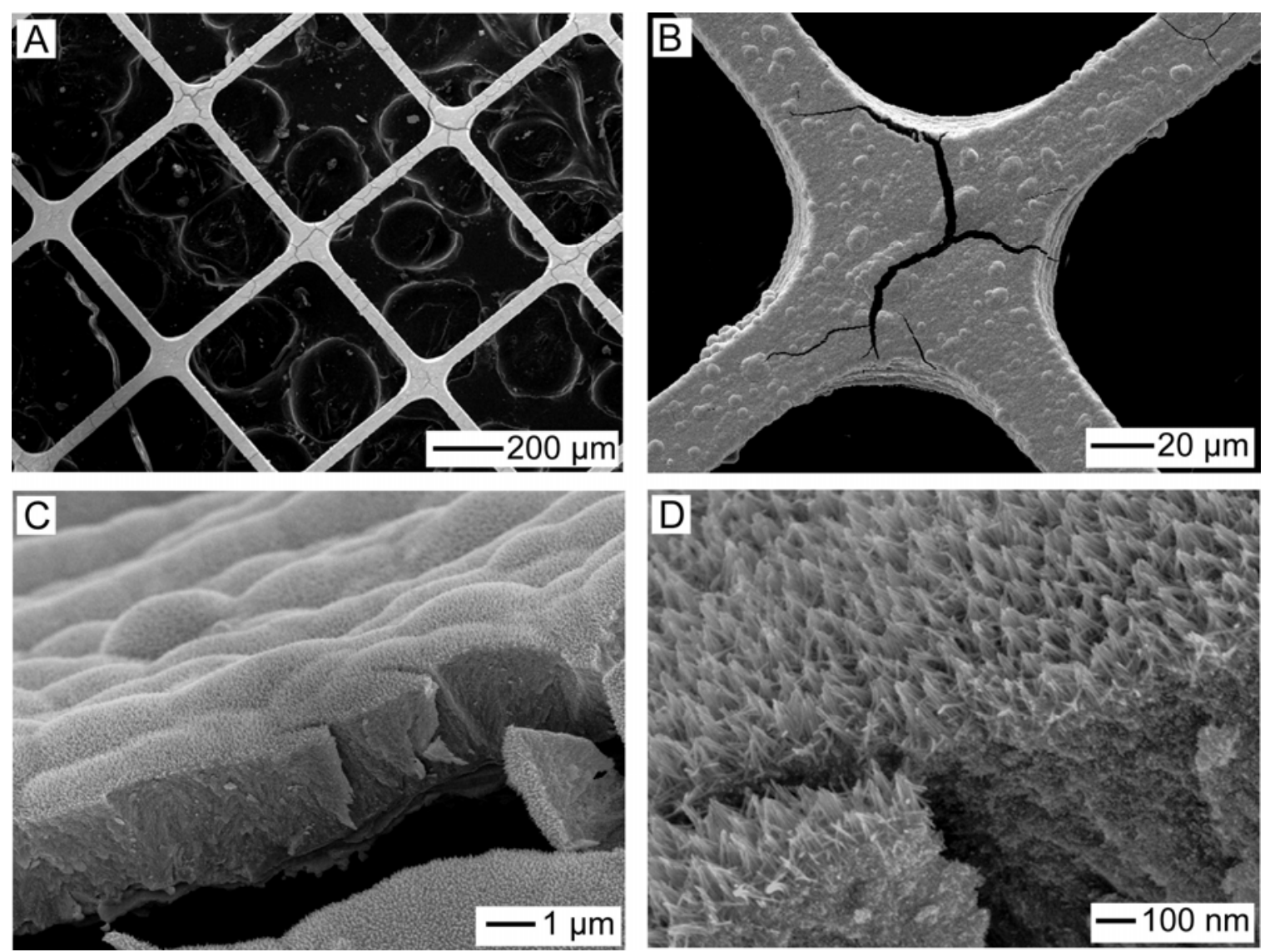

Figure S3. SEM images of Pt nanowires grown directly on the surface of a tungsten gauze. (A, B) An overview of the product at two different magnifications and (C, D) cross-sectional view showing that the Pt nanowires were grown at the very top of a layer composed of aggregated $\mathrm{Pt}$ nanoparticles due to the relatively high concentration of $\mathrm{PtCl}_{6}{ }^{2-}$ used for the synthesis. 


\section{Experimental Procedure}

In a typical procedure, $4 \mathrm{~mL}$ of ethylene glycol (EG, J. T. Baker, Lot\# A34B16) was injected into a 3-neck flask (fitted with a reflux condenser and a Teflon coated stir bar) and heated in air to $110{ }^{\circ} \mathrm{C}$ for $30 \mathrm{~min}$. A Pt gauze $(5 \mathrm{~mm}$ x $5 \mathrm{~mm}$, Alfa Aesar, 100 mesh woven from $0.0762 \mathrm{~mm}$ diameter wire, $99.9 \%$ metals basis) was then added to the ethylene glycol along with $20 \mu \mathrm{L}$ of a 20-mM iron species $\left(\mathrm{FeCl}_{3} \cdot 6 \mathrm{H}_{2} \mathrm{O}\right.$ or $\mathrm{FeCl}_{2} \cdot 6 \mathrm{H}_{2} \mathrm{O}$, Aldrich, pre-dissolved in ethylene glycol). The solution was heated for an additional $30 \mathrm{~min}$ to boil off any trace amount of water. $400 \mathrm{mM}$ poly(vinyl pyrrolidone) (PVP, M.W. $=55,000,0.045 \mathrm{~g}$, Aldrich) and $80 \mathrm{mM} \mathrm{H}_{2} \mathrm{PtCl}_{6}(0.033 \mathrm{~g}$, Aldrich) were dissolved separately in $2 \mathrm{~mL}$ of ethylene glycol at room temperature. These two solutions, each of $1 \mathrm{~mL}$ in volume, were then added simultaneously into the flask over a period of $1.5 \mathrm{~min}$. The molar ratio between $\mathrm{H}_{2} \mathrm{PtCl}_{6}$ and the repeating unit of PVP was 1:5. The reaction mixture was continued with heating at $110^{\circ} \mathrm{C}$ in air. After the reaction had proceeded for $18 \mathrm{~h}$, the final solution was colorless with black aggregates on the Pt gauze and at the bottom of the flask. The Pt gauze was washed thoroughly with ethanol and water to remove ethylene glycol and excess PVP, and characterized by both scanning electron microscopy (SEM) and transmission electron microscopy (TEM).

\section{Instrumentation}

The SEM sample was prepared by placing the washed Pt gauze on a carbon tape, and letting it dry under ambient conditions. SEM images were taken using a Sirion XL field-emission microscope (FEI, Hillsboro, OR) operated at an acceleration voltage in the range of $10-15 \mathrm{kV}$. TEM images were performed using a Philips CM100 microscope with the high-resolution pole piece operated at $100 \mathrm{kV}$. 


\section{Measurements of the Electrochemical Surface Areas of Pristine and Nanowire-Coated Platinum Gauzes}

Sample treatment and characterization: Prior to the measurement, the pristine and nanowirecoated Pt gauzes were thoroughly washed with chloroform, ethanol, and finally distilled water. The electrochemical properties were examined with a CHI 760 dual channel electrochemical workstation ( $\mathrm{CH}$ instruments, Inc.) using a three-electrode system, which consists of a working electrode made of $5 \mathrm{~mm} \times 5 \mathrm{~mm}$ gauze (Alfa Aesar, 100 mesh woven from $0.0762 \mathrm{~mm}$ diameter wire, $99.9 \%$ metals basis) or nanowire-coated gauze connected by a Pt wire, a Pt gauze counter electrode $(100 \times 100 \mathrm{~mm}, 52$ mesh woven from $0.1 \mathrm{~mm}$ diameter wire, $99.9 \%$ metals basis from Alfa Aesar), and an $\mathrm{Ag} / \mathrm{AgCl}$ reference electrode. We used hydrogen adsorption-desorption on platinum in acidic condition to measure the electrochemical surface area (ECSA). The tests were conducted using $0.1 \mathrm{M}$ of perchloric acid $\left(\mathrm{HClO}_{4}\right)$ aqueous solution that was purged with argon for about $20 \mathrm{~min}$. After the purge, a continuous steam of argon was introduced into the cell above the liquid surface to maintain an inert atmosphere over the testing solution. The cyclic voltammetry $(\mathrm{CV})$ was recorded between -0.194 and $0.806 \mathrm{~V}$ vs. $\mathrm{Ag} / \mathrm{AgCl}$ with a scan rate of 1 $\mathrm{mV} / \mathrm{s}$. The ECSA was calculated, based on a conventional method, from the area under a backward potential curve between -0.144 and $0.206 \mathrm{~V}$, the region for hydrogen adsorption (Figure S4) based on the following equations: ${ }^{1-5}$

$$
\begin{aligned}
& \text { Charge }[C]=\int_{t o}^{t 1} I d t=\int_{-0.144}^{0.206} \frac{I[A] \times d E[V]}{v[V / s]} \\
& E C S A\left[\mathrm{Cm}^{2} P t\right]=\frac{\text { charge }[\mu C]}{Q\left[\mu C / \mathrm{cm}^{2} \mathrm{Pt}\right]}
\end{aligned}
$$

where $C$ is charge, $I$ the current, $E$ the potential, $v$ the scan rate, and $Q$ the charge density passed during the hydrogen adsorption. The value of $Q$ used for ECSA calculation was experimentally determined to be $210 \mu \mathrm{C} / \mathrm{cm}^{2}$ for a flat Pt surface, which represents the maximum hydrogen adsorbed and is fairly close to the average value expected for the full coverage of close-packed platinum surfaces. ${ }^{4}$

1. Parthasarathy, A.; Srinivasan, S.; Appleby, A. J.; Martin, C. R., J. Electrochem. Soc. 1992, 139, 25302537.

2. Schmidt, T. J.; Gasteiger, H. A.; Stab, G. D.; Urban, P. M.; Kolb, D. M.; Behm, R. J., J. Electrochem. Soc. 1998, 145, 2354-2358.

3. Sogaard, M.; Odgaard, M.; Skou, E. M., Solid State Ion. 2001, 145, 31-35.

4. Brummer, S. B., J. Phys. Chem. 1965, 69, 562.

5. Trasatti, S.; Petrii, O. A., Pure Appl. Chem. 1991, 63, 711-734. 


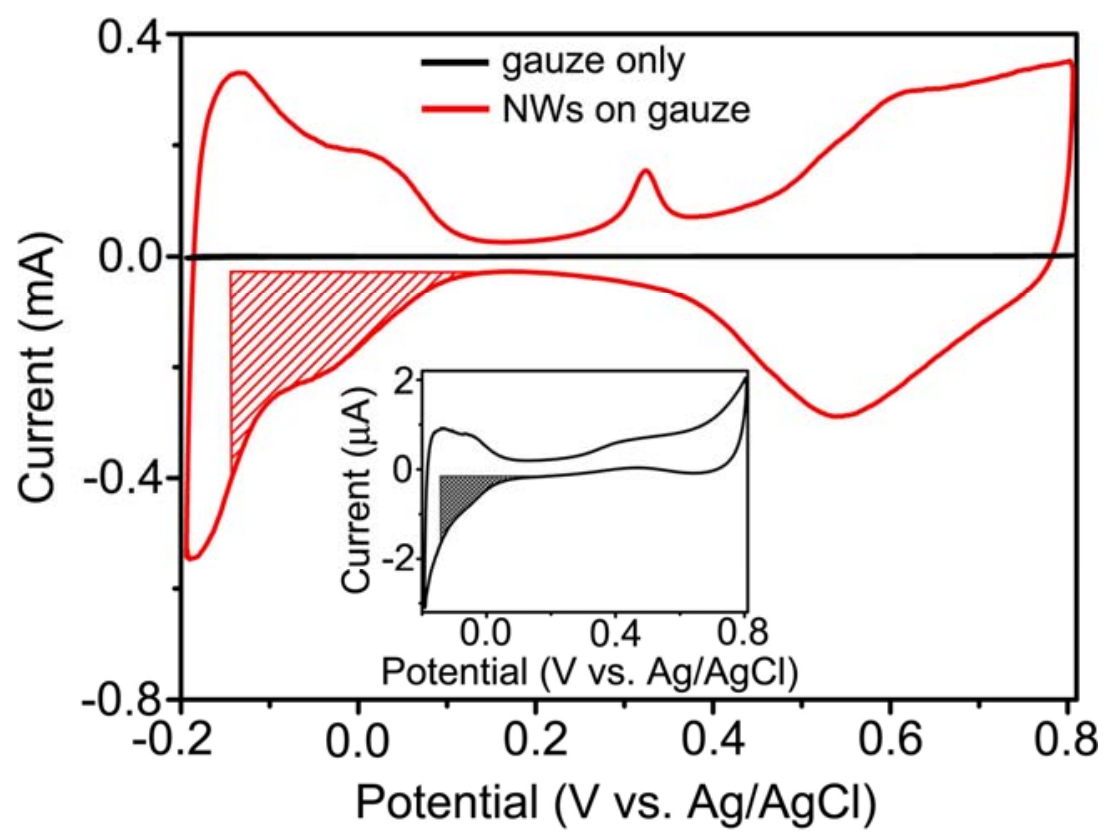

Figure S4. CV curves of pristine (black line, inset) and nanowire (NW)-coated (red line) Pt gauzes for the ECSA measurement. The nanowires were grown under the following conditions: $\mathrm{H}_{2} \mathrm{PtCl}_{6}(80 \mathrm{mM})$, in the presence of PVP $(400 \mathrm{mM})$ and $\mathrm{FeCl}_{3} \cdot 6 \mathrm{H}_{2} \mathrm{O}(20 \mathrm{mM})$.

From these data, we obtained total charges of $3.89 \times 10^{-2} \mathrm{C}$ for nanowires on $5 \mathrm{~mm} \times 5 \mathrm{~mm}$ gauze and $1.32 \times 10^{-4} \mathrm{C}$ for pristine Pt gauze. The corresponding active surface areas were $185 \mathrm{~cm}^{2}$ for the nanowires and $0.62 \mathrm{~cm}^{2}$ for the pristine gauze (close to its calculated geometric surface area of $0.44 \mathrm{~cm}^{2}$ ), giving about two to three orders of magnitude increase in active surface area for nanowire-coated gauze. 